“Transfer” XV: 1-2 (2020), pp. 271-388. ISSN: 1886-554

\title{
LA RECEPCIÓN DEL TEATRO DE FRANCA RAME EN ESPAÑA
}

Milagro Martín Clavijo (ORCID 0000-0001-7300-9493)

Universidad de Salamanca

\section{El teatro de Franca Rame. Problemas de autorialidad o maternidad de sus textos}

Para poder comenzar a analizar la recepción del teatro de Franca Rame en España es necesario partir de una premisa que puede parecer baladí a primera vista: el problema de la autorialidad referido a la producción teatral de Dario Fo y Franca Rame.

Por un lado, Fo es el genio, conocido a nivel mundial ya en los años setenta y, más tarde, en 1997, galardonado con el Premio Nobel de Literatura. Esa fama se traduce en que normalmente los críticos tienden a darle más visibilidad y otorgarle un lugar preferente en sus reseñas o entrevistas dejando a Franca en un perpetuo segundo plano. ${ }^{1}$

Por otro lado, Franca Rame es la mujer ${ }^{2}$ de Dario Fo, llevan viviendo juntos desde los años cincuenta y trabajando codo a codo tanto en la escena como en la escritura de sus obras, de tal manera que realmente podríamos hablar de una escritura a cuatro manos, ${ }^{3}$

\footnotetext{
${ }^{1}$ Un ejempo muy claro lo tenemos cuando en diciembre de 1998 Franca Rame recibe en Madrid el Premio León Felipe 1998 por los Derechos Humanos. Este acontecimiento coincide también con la asistencia de Fo en Valencia para la celebración del 50 aniversario de la Declaración Universal de los Derechos Humanos por parte de la Unesco. Del premio de Franca apenas se habla, Fo se lleva el protagonismo e, incluso en las breves reseñas sobre el premio, se le dedica bastante más espacio a Fo. (Torres,1998).

2 Concetta D'Angeli (2016: 17), como antes Luciana D'Arcangeli y Joseph Farrell, se ha referido a este aspecto en su artículo "Franca Rame: l'ombra" en el que afirma que "per la sua intera esistenza, Franca Rame è stata l'ombra di Dario Fo", considerada por la crítica como mujer de Fo y se le ha negado una autonomía en el ámbito artístico.

${ }^{3}$ De hecho, como afirma Fabio Contu (2017: 221-222), se debería hablar más concretamente de "multiautorialità" o utilizar el concepto de co-autoría.
} 
“Transfer” XV: 1-2 (2020), pp. 271-388. ISSN: 1886-554

algo que en teatro es muy habitual (Contu 2017: 188). Tal y como afirman los dos en reiteradas entrevistas, ${ }^{4}$ prácticamente no se puede hablar de autoría única en el caso de ningún texto y, sobre todo, en los que versan sobre la condición femenina desde finales de los años setenta, y en las décadas de los ochenta y noventa incluso se puede afirmar una mayor implicación en el escritura por parte de Franca.

Sin embargo, fundamentalmente hasta los años noventa, ${ }^{5}$ en sus publicaciones y también en los carteles y los programas de mano de sus representaciones lo habitual es que figure como autor únicamente Dario Fo y el nombre de Franca Rame aparezca solo como intérprete. Únicamente en contadas ocasiones aparece la autoría de los dos. Es el caso de algunas de las obras de cuya recepción en España trataremos en esta sede: aquellas que giran en torno a la mujer ${ }^{6}$ y en las que Franca Rame ha tenido un papel fundamental en la escritura, es decir, en las que podemos hablar de maternidad de Rame y que Fabio Contu elenca tras un estudio pormenorizado: Tutta casa, letto e chiesa (1977-1985), Coppia

\footnotetext{
${ }^{4}$ Fo ha declarado que las obras a partir de Venticinque monologhi di una donna y todos los personajes femeninos han sido escritos por Franca Rame. Por su parte, en una entrevista a Farrell, Rame señala: "Se non fossi stata sua moglie, se fossi stata un'altra, avrebbe dovuto, per forza di cose, mettere anche il mio nome come autrice. Einaudi ha pubblicato numerosi testi di Dario curati da me e inizialmente non figurava nemmeno la dicitura 'a cura di Franca Rame' [...] Il guaio è che io sono la moglie, e la moglie, talvolta, è un mobile di casa, un quadro alla parete, qualcosa che ti sta vicino ma che non vedi. [...] Nei testi di argomento femminile (come ad esempio Tutta casa, letto e chiesa) il mio nome come autrice lo mise... non poteva farne a meno" (Rame 2013: 87-89).

${ }^{5}$ Desde 1999 sus textos teatrales publicados en Italia (por Einaudi y por Fabbri) aparecerán sistemáticamente con la firma de los dos.

6 "Il ruolo di Franca è quello di coautore, oppure di ispiratrice, suggeritrice, revisora, di editor insomma? Una editor che gode di larghissima libertà d'intervento e che ha esercitato un ruolo ancora più attivo nelle pièces 'femministe', dove il suo coinvolgimento emotivo era maggiore, a causa dei temi affrontati, e le motivazioni autobiografiche più forti" (D'Angeli 2016: 23).
} 
“Transfer” XV: 1-2 (2020), pp. 271-388. ISSN: 1886-554

aperta, quasi spalancata (1983), Parliamo di donne (versión del 1991) y Sesso? Grazie, tanto per gradire! (1994). ${ }^{8}$

Por lo que se refiere a la publicación de estas obras en España, hay que destacar, en primer lugar, la importante labor de traducción de Carla Matteini (1939-2013). Esta dramaturga y traductora se ha encargado de la traducción y de la adaptación de la versión en castellano al tipo de representación y a la época de una buena parte de los textos de Fo y Rame en español y ha contribuido fuertemente a su difusión en ámbito hispano. En segundo lugar, Ediciones Júcar es la editorial que más ha apostado por estos textos; Pareja abierta, Ocho monólogos y Un día cualquiera. Tengamos el sexo en paz lo publicó la editorial vasca Hiru Argitaletxa en 1996.

\section{La recepción en España de Tutta casa, letto e chiesa (1977)}

Tutta casa, letto e chiesa está formado por varios monólogos centrados en la condición femenina. Por tanto, desde su primera edición en 1977, en plena lucha del movimiento feminista y en apoyo explícito a esta, hasta la última de 1985 a la que hemos tenido acceso, tanto la estructura de la obra como los proprios monólogos han ido cambiando. Incluso algunos de ellos se han introducido en espectáculos con nombres distintos como Otto monologhi. Dependiendo de la edición podemos encontrarnos con los siguientes monólogos: los más habituales son "Il risveglio", "Una donna sola", "La mamma fricchettona", "Abbiamo tutte la stessa storia", "Medea" y "Lo stupro", pero también aparecen "Monologo della puttana in manicomio", "Alice nel paese senza meraviglie" y "Contrasto per una sola voce".

\footnotetext{
${ }^{7}$ Sobre la recepción en España de Parliamo di donne del 91 y que comprende fundamentalmente dos actos únicos, "La heroína" y "Gordura es hermosura" no he encontrado representaciones en España antes del 2000.

${ }^{8}$ De La scelta di Eva, 2013, no nos ocuparemos en esta sede porque se sale del límite cronológico que nos hemos trazado, los años ochenta y noventa.
} 
“Transfer” XV: 1-2 (2020), pp. 271-388. ISSN: 1886-554

Esto significa que las compañías que han llevado a escena el espectáculo tampoco lo han hecho siempre ni con los mismos monólogos ni con la misma estructura. A esta dificultad inicial a la hora de rastrear su recepción, se añade otra más: el título italiano difícilmente traducible. Por eso, muchas compañías han optado por poner un nombre distinto, a veces tan lejano al original como Orgasmo adulto escapó del zoo, bajo el que Franca Rame representó su espectáculo en el Centro Cultural Villa de Madrid con motivo del IV Festival Internacional de Teatro en abril de 1984. En esta ocasión se señala a Fo como coautor de los textos pero, como veremos en otras reseñas de espectáculos derivados de esta obra y salvo raras ocasiones, el nombre de Franca viene siempre subordinado al de su marido, se la presenta siempre en relación a él, como su mujer, su compañera, se la compara a menudo con él y, generalmente, sale malparada.

En una reseña de $A B C$ del 5 de abril se señala que esta obra presenta muchos aspectos en común con otras de Fo: "Similitudes, pero sin la capacidad gestual del marido, la prodigiosa inventiva, el chispeante ingenio, sus habilidades de artificiero teatral" ("Franca Rame, de la fábula..." 1984). ${ }^{9}$ Además, se recalca su vertiente política ${ }^{10} y$, de manera, especial, su feminismo "de tono victimario": "Moraleja: la mujer es una clase oprimida tanto en el aspecto laboral como en el sexual, en una sociedad donde los hombres hacen las leyes y los hombres las aplican; El tono

\footnotetext{
${ }^{9}$ Algunas de las reseñas que cito en este artículo aparecen sin número de página y, en alguna ocasión, sin día exacto de su publicación. He accedido a esos textos a través del Archivio Franca Rame y, por lo tanto, gracias a los recortes que Fo y Rame conservaron, escanearon y subieron a su archivo on line. Sin embargo, en algunas ocasiones, la imagen escaneada no contiene todos los datos requeridos en una bibliografía académica. Desgraciadamente, no hemos podido acceder directamente a todos esos artículos de periódicos de muchas partes de España y publicados en los años ochenta y noventa para completar los datos que faltaban. ${ }^{10}$ Haro Tecglen (1984) recalca que "su contenido es sobre todo político: es un mitin escenificado". La misma Rame declara durante este Festival: "Soy ante todo una mujer política" (Solís 1984: 3).
} 
“Transfer” XV: 1-2 (2020), pp. 271-388. ISSN: 1886-554

excesivamente vindicativo de los cuadros quita eficacia al producto teatral" ("Franca Rame, de la fábula..." 1984).

En 1982 se lleva a escena en la Sala Olimpia de Madrid Tres monólogos, en los que se incluye "Una mujer sola", "Todas tenemos la misma historia" y "Yo, Ulrike Meinhoff". ${ }^{11}$ El espectáculo lo promueven movimientos feministas y la Librería de las mujeres y los ingresos se destinan a la ayuda a feministas. En esta ocasión se califica a Franca Rame como "luchadora feminista de vanguardia" y se le otorga el mismo título que a Fo, el de "juglaresa, sola en escena, con palabra y gesto" (Haro 1982: 39).

En 1999 La Perversa en co-producción con la Compañía de Teatro Cínico (La Plata, Argentina) estrena en el teatro Malic de Barcelona Dos mujeres, espectáculo interpretado por Anna Sabatè ${ }^{12}$ y dirigido por Jerónimo Casas. El director decide llevar a escena dos mónologos: "La madre pasota" y "La violación". Es curioso cómo se define en la ficha técnica el género del espectáculo: tragicómico de pequeño formato. Como veremos, la dificultad para adjudicar las obras de Fo-Rame a un determinado género tea-tral tradicional es un punto común de muchas de las reseñas en estos años.

En el Teatre Grec de Barcelona, en julio de 1980, se representa La mujer en casa, en la iglesia y en la cama, considerada por Mercedes Rivas (1980) como "una de sus obras más polémicas", pero sin entrar luego en detalles.

Un año más tarde el Colectivo teatral Guirigai lleva al Teatro Valtadolin de Madrid Una mujer sola, tres historias sobre la condición femenina sacadas también de esa misma obra: una

\footnotetext{
${ }^{11}$ Io, Ulrike, grido, 1975, es uno de los tres monólogos sobre terrrorismo y represión junto a Accadde domani, 1977, y Una madre, 1980. Aparece en Tutta casa letto e chiesa, 1975, y mas tarde en Fabulazzo osceno, 1980.

${ }^{12}$ Es interesante notar el interés de esta actriz por los textos de Rame. En 1990 representa La Madre pasota en Vis de Vanadi, Tarragona y recibe el Premio especial de interpretación en la séptima muestra juvenil de teatro otorgado por el Institut Català de Serveis a la Joventut. Siete años más tarde lleva a escena El sexe nostro de cada dia, en el Teatro Metropol de Tarragona y el año siguiente en el Teatro Artenbrut de Barcelona.
} 
“Transfer” XV: 1-2 (2020), pp. 271-388. ISSN: 1886-554

joven esposa trabajadora, un ama de casa agobiada por la soledad y una madre que busca a su hijo. El espectáculo cuenta con la interpretación de Antonio Bueno, Pilar Marco, Carmen Mesa, Pablo Esteban y Roberto Álvarez, con escenografía de Pablo Esteban y adaptación y dirección de Agustín Iglesias. Desde la compañía se señala el porqué de esa elección: "Hemos seleccionado entre todos los textos que componen Tutta casa letto e chiesa los tres que consideramos más entroncados con nuestra realidad española" ("Montaje de textos..." 1981) y se afirma que "Darío Fo y Franca Rame, así como La Comuna de Milán, son el brillante resultado de una tradición actoral europea, donde denuncia, juego, comicidad y burla se dan la mano para recorrer un camino poco usual" (“Montaje de textos..." 1981).

\section{La recepción en España de Coppia aperta, quasi spalancata} (1983)

Este acto único se llevó a escena por primera vez en 1983 y es la obra de Fo y Rame más representada en el mundo, con más de 600 ediciones. De hecho, en España nos encontramos con un espectáculo ya en octubre de 1984: Pareja abierta, casi de par en par. La obra se representó en el Teatro Bellas Artes de Madrid bajo la dirección Emilio Hernández y con la interpretación de Magüi Mira y Ángel de Andrés. En este caso, como en la mayoría de las obras de Fo-Rame representadas en España, se parte de la versión castellana de Carla Matteini, considerada una "adecuación perfecta al lenguaje coloquial de aquí y ahora" (Arroyo 1984a).

También con esta representación nos volvemos a encontrar con problemas de autoría: en algunas reseñas se señala a Dario Fo como autor y luego en letra pequeña aparece también como autora Franca Rame ("Pareja abierta sátira" 1984: 62), en otras se habla de la colaboración de Fo con su mujer (Bejarano 1984; Avilés 1984), aunque en esta última los halagos sean todos para Fo. De cual- 
“Transfer” XV: 1-2 (2020), pp. 271-388. ISSN: 1886-554

quiera de las maneras en el programa de sala sí que aparece la coautoría.

A la hora de presentar el género, de nuevo los recensores se encuentran con problemas para definirlo: "No es teatro puro. Es una mezcla de relato y de acción, o mejor es un relato accionado. Participa pues de lo épico y de lo dramático ("Pareja abierta, sátira” 1984: 62).

Con respecto a la valoración conjunta del espectáculo, por un lado, algunos recensores alaban la "construcción perfecta del diálogo, agilidad y fluidez, que permite al actor llegar a una especie de catarsis informal e histriónica" (Avilés 1984: 31), y el hecho de que sea "un alegato social, muy vivaz, lleno de ingenio y verdad profunda. Algo así como una patada en la espinilla de los sacerdotes progres de la libertad sexual" ("Pareja abierta, sátira" 1984: 62). Pero, por otro, consideran que esta obra tiene "menos entidad y cuerpo que las anteriores de Fo-Rame" (Bejarano 1984) y que "a pesar de esa libertad de acción que dejan las obras de Fo sus personajes son modelos definidos y poco variados proclives a la peripecia, la exageración y la excesiva gestualidad" (Avilés 1984: 31).

En 1988 el Gat (Grup d'Acció Teatral) presenta en el Teatro Condal de Barcelona Joc de dos con Carme Sansa y Enric Majó, dirección de Enric Flores y adaptación y realización de Quim Vilar. En esta ocasión se hace hincapié en la autoría del espectáculo: está escrita por Dario Fo en colaboración con su mujer, aunque luego se reconozca: "Es una de esas obras firmadas a due por los dos grandes actores, autores, directores, bestias escénicas del teatro italiano" (Pérez de Olaguer 1988).

En este espectáculo en catalán el título cambia completamente con respecto al italiano: Joc de dos. La posible explicación la presenta Joan Anton Benach:

Dario Fo y Franca Rame podían referirse en el título más al asunto de su comedia, por cuanto su estilo de trabajo y sus compromisos profesionales eran sobradamente definidos. Es decir, "vendidos" 
“Transfer” XV: 1-2 (2020), pp. 271-388. ISSN: 1886-554

de antemano. En la imprecisión en cambio de Joc de dos, cabe, evidentemente, todo ("Joc de dos. Programa de mano" 1988).

Como a menudo sucede con las obras en las que es determinante el papel de escritora de Franca Rame, recensores, directores y actores hablan de feminismo en un sentido o en otro, dejando a Dario Fo, coautor, al margen de esta cuestión..$^{13}$ A Rame, se la define como "militante feminista" (Pérez de Olaguer 1988a) y poco después se añade: "No es decisivo el claro matiz feminista que Franca Rame incluye en el espectáculo, aunque sea, eso sí, de agradecer" (Pérez de Olaguer 1988a). Gonzalo Pérez Olaguer (1988b: 59) subraya que se trata de

un texto feminista, aunque tocado con las ya conocidas, divertidas y punzantes ironías y formas de actor, autor y director italiano. Fo Y Rame -y aquí se nota la mano poderosa de la autoraescriben en función de la mujer y apuestan decididamente por ella; el hombre es, abiertamente, ridiculizado. (Pérez Olaguer 1988b: 59)

Pero cuando se habla de feminismo, no todo el mundo lo tiene tan claro. La actriz Carme Sansa subraya que "en absoluto se puede hablar de una obra militante a favor del feminismo, aunque sí la veo como una obra escrita a favor de la mujer" (Pérez de Olaguer, 1988a). Incluso Majó se atreve a separar los roles de cada uno de los autores: "la militancia de Franca Rame con el gran humor de Dario Fo" (Pérez de Olaguer 1988a), eso sí, en sabio equilibrio.

Joan Sagarra (1988) no se muestra demasiado entusiasta con la obra: la califica de "pochade feminista, con alguna que otra referencia a Muerte accidental de un anarquista y, al final, un sarcástico homenaje a Goldfinger". A pesar de todo, concluye que se trata de un "espectáculo bien construido, bien resuelto, llevado

${ }^{13}$ Solo Quim afirma que la pièce "se mueve en la misma línea de teatro militante y locamente divertido de toda la obra conocida de Dario Fo" (Pérez de Olaguer 1988a). 
“Transfer” XV: 1-2 (2020), pp. 271-388. ISSN: 1886-554

a buen ritmo, con una interpretación brillante, en el que el público se ríe y sale la mar de contento" (Sagarra 1988).

En la misma tónica, Pérez Olaguer (1988b: 59). considera que este texto tiene la impronta clara de Dario Fo, aunque no será una de las obras más importantes "y más propia, diría, de un teatro de la inmediatez. Y, como tal, efectista y efectiva".

Ya a mediados de los años noventa, producciones La Clack lleva a escena Pareja abierta, bajo la dirección de Raquel Toledo. En el programa de sala de 1995 se avisa de que se trata de una versión distinta a la utilizada en las primeras representaciones en español: "se moderniza, pierde un poco de su carga político-ideológica y gana en actualidad, retomando la eterna paradoja de la pareja" ("Pareja abierta. Programa de mano" 1995).

En 1999 se trae de nuevo a escena esta obra bajo el título Una parella oberta, con la dirección de Óscar Molina e interpretada por Iraida Sardà y Joan Cusò. En este caso la adaptación (necesaria ya que el paso del tiempo había hecho evejecer las referencias políticas) es de Ever Martin Banchet. Un montaje nuevo en el que, con palabras de Molina, "es más importante el contenido social, íntimo y el concepto de libertad”. (Ginart 1999).

\section{La recepción en España de Sesso? Grazie, tanto per gradire! (1994)}

Esta obra ha sido definida por los autores como una "conferenzaspettacolo", y parte del libro publicado por Jacopo, el hijo de Franca y Dario, Lo Zen e l'arte di scopare, 1993.

En 1996 esta obra se adapta al español, se representa por primera vez en castellano y en una gira por toda España con el títuloTengamos el sexo en paz. El director es José Carlos Plaza, la actriz Charo López, la producción de Fila Siete y la traducción y adaptación de Carla Matteini. ${ }^{14}$ De los espectáculos de los que nos

\footnotetext{
${ }^{14}$ En varias reseñas se señala la gran traducción y adaptación llevada a cabo por Carla Matteini (Arco, 1996). "Carla Matteini y el director José Carlos Plaza han sido
} 
“Transfer” XV: 1-2 (2020), pp. 271-388. ISSN: 1886-554

ocupamos es probablemente el que más éxito ha tenido en España y que cuenta com más representaciones y más atención por parte de los críticos, entre otros motivos, por la fama de la atriz que lo ha representado. En algunas de las reseñas Charo López se atribuye la idea de traer la pièce a España al verla en Italia y comenta las dificultades iniciales para llevarla a cabo (Adell 1996).

Sobre la cuestión de autoría, en las reseñas de este espectáculo a las que hemos tenido acceso predominan las que hablan de coautoría (aunque luego citen solo a Franca): Alonso (1996) habla de autoría solo de Franca Rame y otros, como Antonio Rodríguez (1996: 75), mencionan única y exclusivamente a Dario Fo.

En términos generales las reseñas suelen ser positivas, tanto para los autores como, sobre todo, para la actriz, Charo López, sobre la que se centran muchos artículos, dejando, a veces, en segundo plano el espectáculo. Por eso, normalmente son las palabras de la actriz las que comentan los reseñadores, pero también, en ocasiones, las de la propia Franca Rame, sobre todo para resaltar la necesidad de hablar de sexo y de acabar con los tabúes en este campo: "tenemos miedo hasta de las palabras. Nuestra sociedad no enseña a amar, ni con el cuerpo ni con el alma. En este espectáculo los sentimientos están en primer plano" (Barbón 1996).

Evidentemente, hablar de sexo, y hacerlo además en un teatro, comportaba una serie de riesgos que podían haber desembocado en un escándalo, ${ }^{15}$ como apuntan distintos críticos como

los encargados de darle a la versión española la credibilidad suficiente en un contexto cultural diferente al italiano" (Luna 1996). Sobre la adaptación comenta Charo López: "Adaptarla ha sido difícil porque en España e Italia no pasan las mismas cosas, ni siquiera en el sentido sexual. En este país sí que ha habido un cambio sustancial en todo lo que se refiere al sexo, han desaparecido las preocupaciones y el miedo, comenta Charo, quien atribuye estas disparidades en la conducta sexual a que los católicos italianos tienen al Papa muy cerca." (Gea 1996). ${ }^{15}$ Como efectivamente había ocurrido en Italia. De hecho, Charo López afirma que "tenía mucho miedo porque en Italia vi lanzar panfletos contra el montaje" ("En España ha habido..." 1996: 35). 
“Transfer” XV: 1-2 (2020), pp. 271-388. ISSN: 1886-554

Antonio Arco (1996) que señala que se trata de "una función de izquierdas" y, por tanto, "se precisa de una cierta dosis de tolerancia para que su seguimiento, su disfrute, no altere la paciencia ni las conciencias de los rígidos de corazón y los posicionados en la intransigencia". Ese riesgo era uno de los grandes miedos de la actriz en las primeras representaciones: con el tiempo afirmará que la situación española es muy distinta, que "en España ha habido una verdadera transición en materia de sexo. No hay escándalo. La gente más conservadora acepta bien el contenido de la obra, porque enseguida se da cuenta que carece de picardía, maldad y dobles sentidos" (“En España ha habido..” 1996: 35).

Salvados esos riesgos, en las recensiones se pone énfasis en la diversión: "es una pura fiesta, un vehículo de diversión" (Arco, 1996), una "alegre operación dialéctica" ("Charo López: Soy la actriz..." 1996) y en el espíritu didáctico del espectáculo. En este sentido, se debate mucho, de nuevo, sobre el género de Tengamos el sexo en paz: "una peculiar mezcla de monólogo dramático y conferencia", ${ }^{16}$ un "ejercicio dramático de difícil catalogación" (Herrero 1996) que desconcierta, pero también estimula al espectador, porque "un monólogo planteado como una lección sobre sexualidad aplicada es una apuesta teatral difícil" (Gil 1996). la obra:

Roberto Herrero (1996) acompaña al público a lo largo de

Al principio de esta obra uno tiene la sensación de acudir a una charla cualquiera de una caja de ahorros cualquiera. Una disertación didáctica, distendida, progre y un poco osada sobre el sexo y sus misterios, especialmente sobre estos últimos. Ante nosotros la profesora-amiga y la confidente [...] Esto no está muy claro si es teatro o conferencia, lección o curso de extensión universitaria.

\footnotetext{
${ }^{16}$ José Ferrandiz Casares (1996) comenta que "evidentemente, es una conferencia, pero nunca debiera olvidarse que la pronuncia una actriz".
} 
“Transfer” XV: 1-2 (2020), pp. 271-388. ISSN: 1886-554

Por suerte está Charo López al mando de la nave y nosotros encantados de ir a clase.

Carlos Gil (1996) se centra en los dos aspectos más importantes que hacen factible sobre la escena una lección sobre sexualidad:

\begin{abstract}
Se requiere dos factores imprescindibles: un texto donde la inteligencia y los guiños dramatúrgicos sirvan de soporte a un hilo conductor preñado de ironía, sentimientos y consejos casi de servicio de salud y alguien que lo interprete con convencimiento ideológico y solvencia artística. Los dos se dan en este trabajo. El texto tiene toda la sabiduría de Rame y Fo, la dosificación de los pasajes, el ritmo interno suficiente para convertir algo que podría ser retórico e incluso provoque un rechazo entre sectores del público al hablar de cosas que se dan por supuestas o por conocidas y que no se ahorran pelos ni señales con un lenguaje que muchas veces llega a ser crudo, pero siempre tamizado con esa pátina de humor corrosivo que provoca la complicidad. (Gil 1996)
\end{abstract}

Sin embargo, algunos de los críticos no están tan contentos con el espectáculo. Nacho Artime (1996: 10) lo considera "un pretexto para hacernos unas risas, con cuatro chistes verdes de cuando éramos colegiales", lo define como un texto "tonto" y expresa su duda "de que un texto tan pretendidamente femenino y feminista [sea] de un machismo que atufa. Solo queda la esperanza de que Charo Lopez estrene pronto una obra de teatro, teatro".

Pedro Manuel Vílora (1997) lo considera un proyecto simpático, pero que no alcanza el resultado de las mejores obras de Fo-Rame:

El texto da bandazos facilones por distintos temas sin centrarse en ninguno, sin desarrollar un argumento sólido, y sin profundizar. Todo queda en una sucesión de anécdotas y avisos para navegantes, que surgen directamente de los estilemas libertarios, 
“Transfer” XV: 1-2 (2020), pp. 271-388. ISSN: 1886-554

contestarios y progresistas de hace veinte y treinta años. El humor y la tendencia ocasional a la caricatura no esconden cierta propensión a lo didáctico, fruto de la desconfianza en el nivel de información del público y lectores que está en el origen mismo de este monólogo.

Solo Charo y Rame, con su brillantez como actrices, "justifican el acercamiento a un texto tan menor" (Vílora, 1997).

Para terminar, en algunas reseñas no se puede evitar la asociación de este espectáculo con el programa de televisión Hablemos de sexo, emitido por la primera cadena de TVE en 1990 y presentado por la sexóloga Elena Ochoa. Con este programa se hablaba en la pequeña pantalla por primera vez en España de temas relacionados con la sexualidad desde un punto de vista científico. En este sentido, la reseña de Sonia Sánchez, en la que compara a la protagonista con la doctora Ochoa "para sacar a la luz cuestiones íntimas" (Sánchez 1996) o también la de Nacho Artime (1996: 10).

Dos años más tarde Haro Tecglen (1998) reseña el espectáculo protagonizado por Charo López en el Teatro Lara de Madrid, da las gracias a los libertarios, como Fo y Rame, que han luchado por una libertad sexual que es básica: "para los que vivimos en las sociedades represoras, esta descarga es sana. Y puede incitar a mayor libertad. O por lo menos a gozar de la que se tiene sin padecer miedo interno".

Este mismo año el grupo de teatro Trono Villegas lleva a escena en catalán El sexe nostre de cada día, en el Jove Teatre Regina. En este caso no se ha representado como un monólogo sino en forma de diálogo entre un hombre y cinco mujeres.

\section{Conclusiones}

Franca Rame y Dario Fo han tenido una considerable recepción en España en los años ochenta y noventa: se han publicado y se han llevado a la escena muchas de sus obras, fundamentalmente, pero 
“Transfer” XV: 1-2 (2020), pp. 271-388. ISSN: 1886-554

no solo, en teatros de Madrid y de Barcelona. ${ }^{17}$ También lo han tenido las obras centradas en la condición de la mujer en las que la mano de Rame es evidente.

Al analizar las reseñas de las representaciones de Tutta casa, letto e chiesa, Coppia aperta, quasi spalancata y Sesso? Grazie, tanto per gradire!, hemos podido comprobar que, en España como en Italia y, a pesar de que ya desde finales de los años noventa las obras de la pareja Fo-Rame se publican con el nombre de los dos, es decir, como co-autores, algunos de los críticos siguen obviando el nombre de Franca o lo subordinan al de Fo, presentándola como colaboradora de unos textos en los que, como hemos visto, la maternidad de Rame es evidente a pesar de estar escritos a cuatro manos. Esto es fundamentalmente evidente en los títulos de las reseñas en los que el nombre de Fo "vende" más que el de Rame.

Por otro lado, a pesar de que las críticas suelen ser positivas, se nota un cierto recelo en algunas reseñas a la hora de afrontar temas, hasta hace poco tabú, como el del sexo o la violencia doméstica, y hacerlo desde el punto de vista de las mujeres. El término feminismo en algunas ocasiones se considera un aspecto negativo del espectáculo y se relaciona solo con Rame.

En tercer lugar, los críticos se interesan por el género teatral al que pertenecen estos monólogos e intentan, con más o menos atino, explicárselo a los lectores.

\section{Referencias bibliográficas}

ADELL, Xavier. (1996). "Charo López: a través del sexo podemos ser mejores". El Periódico de Catalunya 19 de marzo.

ALONSO, Andoni. (1996). "Charo López: nunca pensé que yo podría estar sola sobre un escenario”, El Mundo del País Vasco.

\footnotetext{
${ }^{17}$ Para la recepción en España de la obra de Fo y Rame hasta la concesión del Nobel, véase Martín Clavijo 2016.
} 
“Transfer” XV: 1-2 (2020), pp. 271-388. ISSN: 1886-554

Disponible en: $<<$ http://www.archivio.francarame.it/ scheda.aspx?IDScheda $=16861 \& I D O p e r a=159>>$ [Última consulta $12 / 3 / 2019]$.

ARCHIVIO FRANCA RAME (s.d.). Disponible en: $<<$ http://www.archivio.francarame.it/ $>>$ [Última consulta $10 / 3 / 2019]$.

ARCO, Antonio. (1996). "Tengamos el sexo en paz. Charo López". La Verdad. <<http://www.archivio.francarame.it/ $\underline{\text { scheda.aspx?IDScheda=16861\&IDOpera }=159>>\text { [Última consulta }}$ $13 / 3 / 2019]$.

ARROYO, Julia. (1984). "Pareja abierta. Peripecias conyugales". Madrid. Disponible en:

$<<$ http://www.archivio.francarame.it/scheda.aspx?IDScheda $=17$ 737\&IDOpera $=47>>$ [Última consulta 1/3/2019].

---. (1984b). "IV Festival Internacional de Teatro. Denuncia feminista". Ya, 5 abril, .

ARTIME, Nacho. (1996). "El sexo a risa”. La Nueva España, p. 10. Disponible en: $<<$ http://www.archivio.francarame.it/scheda.aspx?IDScheda=16 861\&IDOpera=159>> [Última consulta 14/3/2019].

AVILÉS, Juan C. (1984). "Pareja abierta, casi de par en par, en el Bellas Artes. Fo, siempre a flote”. Guía del ocio 10/9/8, p. 31.

BARBÓN, Marta. (1996). "Charo López estrena hoy en el teatro Jovellanos Tengamos el sexo en paz". La voz de Asturias 5 de marzo.

BEJARANO, Fernando. (1984). "Magüi Mira y Ángel de Andrés, una divertida pareja abierta". Disponible en:

$<<\mathrm{http}: / /$ www.archivio.francarame.it/scheda.aspx?IDScheda $=17$ 737\&IDOpera $=47>>$ [Última consulta 2/3/2019].

CONTU, F. (2017). Zona Franca (Rame). Tesis doctoral. Sevilla: Universidad de Sevilla, dirigida por Mercedes Arriaga Flórez y Milagro Martín Clavijo.

D’ANGELI, Concetta. (2016). "Franca Rame: l'ombra”. En: CERRATO, Daniele (ed.). Franca, pensaci tu. Studi critici su Franca Rame. Roma: Aracne, pp. 17-30. 
“Transfer” XV: 1-2 (2020), pp. 271-388. ISSN: 1886-554

FERRANDIZ CASARES, José. (1996). "Charo López batalla en solitario”, El Información ,24 marzo.

FO, Dario; Rame, Franca\& Fo, Jacopo. (1996). Tengamos el sexo en paz. Hondarribia: Hiru Argitaletxa.

GEA, Juan Carlos. (1996). "Charo López: quiero desmitificar con humor los tabúes sexuales”, La Nueva España, 6 de marzo.

GIL, Carlos. (1996). "Una actitud solidaria”, Egin 4 mayo.

GINAR, Belén. (1999). "Dario Fo y la pareja”, El País 28 noviembre.

HARO TECGLEN, Eduardo. (1982). Franca Rame, una luchadora de vanguardia”, El País 15 noviembre, p. 39.

---. (1984). "Mitin en escena. Orgasmo adulto escapado del zo", El País 6 abril.

--. (1998). "El sexo según Fo. Tengamos el sexo en paz", El País 16 febrero.

HERRERO, Roberto. (1996). "Encantados de ir a clase", Diario Vasco, 9 marzo.

LUNA, Antonio Jesús. (1996). "Charo López habla de sexo. El Gongora acoge”, Diario de Córdoba, 15 marzo.

MARTÍN CLAVIJO, Milagro. (2016). "El teatro de Dario Fo y Franca Rame en España hasta la concesión del Nobel”, Transfer XI (1-2) mayo: 165-186.

[Última consulta 4/3/2019].

PÉREZ DE OLAGUER, Gonzalo. (1988a). "Enric Majó y Carme Sansa, en una obra sobre la pareja" El Periódico. Disponible en: $<<$ http://www.archivio.francarame.it/scheda.aspx?IDScheda $=17$ 748\&IDOpera $=47>>$ [Última consulta 6/3/2019].

---. (1988b). "Esta vez, la mujer es la que se impone", El Periódico, 12 noviembre, p.59.

RAME, Franca. (2013). Non è tempo di nostalgia, con Joseph Farrell. Pisa-Cagliari: Della Porta editori.

RAME, Franca \& Fo, Dario (1988). Un día cualquiera. Madrid: Ediciones Júcar.

---. (1990). Ocho monólogos. Madrid: Ediciones Júcar.

---. (1986/8). Pareja abierta (casi de par en par). Madrid: Ediciones Júcar. 
“Transfer” XV: 1-2 (2020), pp. 271-388. ISSN: 1886-554

RIVAS, Mercedes. (1980). "Presentación en Barcelona de dos espectáculos teatrales de Dario Fo",El País ,11 julio.

RODRÍGUEZ, Antonio. (1996). "Los treinta años de mi vida se cristalizan en esta obra", Diario de Córdoba 16 marzo, p. 75.

S.A. (1981). "Montaje de textos de Darío Fo y Franca Rame sobre la condición de la mujer", El País, 15 diciembre,

S.A. (1984). "Pareja abierta, sátira sexual de Dario Fo", $A B C$, p. 62. Disponible en:

$<<$ http://www.archivio.francarame.it/scheda.aspx?IDScheda $=17$ 737\&IDOpera $=47>>$ [Última consulta 7/3/2019].

S. A. (1984). "Franca Rame, de la fábula feminista al discurso político”, $A B C, 5$ abril.

S. A. (1988). "Joc de dos. Programa de mano".

S. A. (1995). "Pareja abierta. Programa de mano".

S. A. (1996). "En España ha habido una verdadera transición en materia de sexo", Heraldo de Aragón, 21 mayo, p. 35.

S. A. (1996). "Charo López: Soy la actriz más plena y gratificada", El correo gallego, 27 de febrero.

SAGARRA, Joan de. (1988). "Una pochade a la catalana, Joc de dos (Coppia aperta, quasi spalancata)", El País, 13 noviembre.

SÁNCHEZ, Sonia. (1996). "Charo López remueve los cimientos del sexo", Diario 16, 13 marzo.

SOLÍS, Silvia. (1984). "Soy ante todo una mujer política", Pueblo, jueves 5 abril, p.3.

TORRES, Rosana. (1998). "El Nobel visita España con su mujer, Franca Rame, que recibe el Premio León Felipe”, El País, 10 diciembre.

VÍLORA, Pedro Manuel. (1997). Tengamos el sexo en paz, ABC literario, 17 octubre.

Fecha de recepción: 2.06.2019 Fecha de aceptación: 01.07.2019 
“Transfer” XV: 1-2 (2020), pp. 271-388. ISSN: 1886-554

\title{
Resumen:
}

En este artículo se pretende estudiar la recepción de la obra y de las representaciones de Franca Rame en España durante los años ochenta y noventa. Fuentes del estudio las constituyen reseñas, artículos, reportajes y entrevistas publicadas en España en este periodo a raíz de espectáculos de los que Rame es autora. Se tendrán en consideración fundamentalmente los aspectos teatrales de su producción, pero sin dejar al margen las consideraciones políticas y morales que de ella derivan.

Palabras clave: Franca Rame, Teatro, España, Traducciones, Representaciones.

\section{THE RECEPTION OF FRANCA RAME'S THEATER IN SPAIN}

\begin{abstract}
:
In this article it will be studied the reception of Franca Rame's theatre plays and performances in Spain, during the eighties and the nineties. Sources of the study are reviews, articles, reports and interviews published in Spain in these decades. The theatrical aspects of her production will be taken into consideration mainly, but without leaving aside the political and moral considerations derived.
\end{abstract}

Keywords: Franca Rame, Theatre, Spain, Translations, Performances. 